\title{
基于软硬酸碱理论的单分散中重稀土硫氧化物纳米板的可控合成
}

\author{
顾均 丁祎柯俊张亚文* 严纯华* \\ (稀土材料化学及应用国家重点实验室 北京大学-香港大学稀土材料和生物无机化学联合实验室 \\ 北京大学化学与分子工程学院 北京分子科学国家实验室 北京 100871)
}

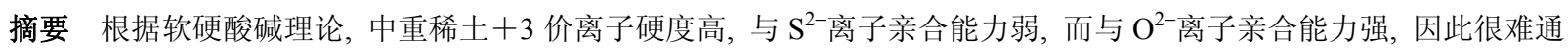
过基于高沸点有机溶剂的前驱体热分解法合成中重稀土硫氧化物纳米材料. 发展了制备掺杂或非掺杂的中重稀土(Eu, $\mathrm{Gd}, \mathrm{Er}, \mathrm{Y}, \mathrm{Yb}$ )的单分散硫氧化物纳米板的前驱体热分解方法. 密度泛函理论计算表明, 从轻稀土元素到中重稀土元素, 合成硫氧化物所需要的硫元素化学势逐渐升高. 实验结果表明: 在油胺中以 $\mathrm{H}_{2} \mathrm{~S}$ 气体为硫化试剂, 以分解温度低的稀 土乙酰丙酮盐为前驱体在 $280{ }^{\circ} \mathrm{C}$ 条件下反应, 可以有效提高反应体系中硫元素的化学势, 从而利于目标硫氧化物纳米 晶的生成. 其中, 所获得的 $\mathrm{Y}_{2} \mathrm{O}_{2} \mathrm{~S}$ : Eu 纳米板在 $251 \mathrm{~nm}$ 紫外光激发下可发出红色荧光; 而与体相材料相比, 因其表面原 子比例很高且晶化度较低, 导致其荧光寿命较短、量子产率较低.
\end{abstract}

关键词＼cjkstart稀土硫氧化物; 纳米材料; 液相合成; 密度泛函理论计算; 苂光

\section{Controllable Synthesis of Monodispersed Middle and Heavy Rare Earth Oxysulfide Nanoplates Based on the Principles of HSAB Theory}

\author{
Gu, Jun Ding, Yi Ke, Jun Zhang, Yawen* Yan, Chunhua* \\ (Beijing National Laboratory for Molecular Science, State Key Laboratory of Rare Earth Materials \\ Chemistry and Applications PKU-HKU Joint Laboratory in Rare Earth Materials and Bioinorganic \\ Chemistry, College of Chemistry and Molecular Engineering, Peking University, Beijing 100871, China)
}

\begin{abstract}
Based on the theory of hard and soft acids and bases, trivalent ions of middle and heavy rare earths belong to very hard acids, which possess weak affinity towards $\mathrm{S}^{2-}$ ions but strong affinity to $\mathrm{O}^{2-}$ ions. So it is difficult to synthesize middle and heavy rare earth oxysulfide nano-materials through the thermolysis method in high-boiling-point organic solvent. In this article, monodispersed oxysulfide nanoplates of $\mathrm{Y}, \mathrm{Eu}, \mathrm{Gd}, \mathrm{Er}$ and $\mathrm{Yb}$ were synthesized through this thermolysis method we developed. Both sodium-doped and undoped rare earth oxysulfide nanoplates could be prepared, and the doping of sodium ions could promote the crystallization of the nanoplates. Rare earth acetylacetonates were used as metal precursors and $\mathrm{H}_{2} \mathrm{~S}$ gas as the sulfurizing reagent. The reactions were conducted in oleylamine at $280{ }^{\circ} \mathrm{C}$ for 1 hour. The thermogravimetric analysis of the precursor showed that the initial decomposition temperature of the rare earth acetylacetonates is about $200{ }^{\circ} \mathrm{C}$, which is much lower than that of rare earth oleates. The transmission electron microscopy observation and energy dispersive $\mathrm{X}$-ray analyses of the intermediate products during the synthesis of the nanoplates showed that rare earth oxide nanoplates formed firstly at about $220{ }^{\circ} \mathrm{C}$, and these nanoplates transformed to oxysulfide nanoplates gradually during the temperature ramping period. Density functional theory calculation was used to compare the total free energy of the oxide and oxysulfide of different rare earth elements. According to this thermodynamical comparison, we concluded that, from light rare earths to heavy rare earths, higher chemical potential of sulfur is needed to obtain the oxysulfide rather than oxide. On one hand, $\mathrm{H}_{2} \mathrm{~S}$ gas has higher sulphurizing power than sulfur. On the other hand, a comparatively low reaction temperature favors the dissolving of $\mathrm{H}_{2} \mathrm{~S}$ in oleylamine. As a result, the chemical potential of sulfur in synthetic system could be effectively increased by using rare earth acetylacetonates as the precursors instead of rare earth oleates, and using $\mathrm{H}_{2} \mathrm{~S}$ gas as sulphurizing agent instead of sulfur, which made it possible to prepare middle and heavy rare earth oxysulfide nanoplates in oleylamine. Fluorescent measurements showed that as-synthesized $\mathrm{Y}_{2} \mathrm{O}_{2} \mathrm{~S}$ :Eu nanoplates could emit red light under $251 \mathrm{~nm}$ ultraviolet light excitation. For $\mathrm{Y}_{2} \mathrm{O}_{2} \mathrm{~S}$ :Eu nanoplates, the fluorescence life time was shorter and quantum yield was lower in comparison with the corresponding bulk counterpart, possibly due to its much higher portion of surface atoms as well as lower crystallinity. Keywords rare earth oxysulfide; nanomaterial; liquid synthesis; density functional theory calculation; fluorescence
\end{abstract}

1 引言

稀土元素(rare earth, RE)所独有的 4f 电子结构赋予
了其独特的光学和磁学性质 ${ }^{[15]}$, 因此，稀土基纳米材

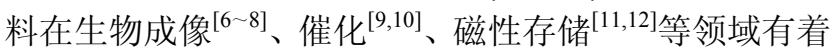
广阔的应用前景. 目前, 人们已经通过溶液相的合成方

*E-mail: ywzhang@pku.edu.cn; yan@pku.edu.cn

Received December 7, 2012; published January 9, 2013.

Supporting information for this article is available free of charge via the Internet at http://sioc-journal.cn.

Project supported by the National Natural Science Foundation of China (Nos. 21025101, 21271011).

项目受国家自然科学基金(Nos. 21025101, 21271011)资助. 
法, 得到了多种不同类型的稀土基纳米材料, 这其中包

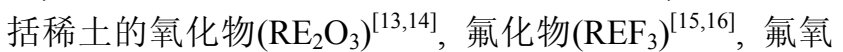
化物 $(\mathrm{REOF})^{[17]}, \mathrm{NaREF}_{4}$ 型氟化物复盐 ${ }^{[18 ~ 21]}$, 氯氧化物

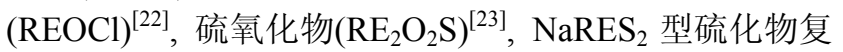
盐 ${ }^{[24]}$ 等. 在已发展出的合成方法中, 一类重要方法是高 沸点有机溶剂中的前驱体热分解法. 用该方法可以合成 出多种尺寸和形貌可控、晶化度高、单分散性好的稀土 化合物纳米晶. 根据软硬酸碱理论, 稀土 +3 价离子由 于其电荷高而半径小, 属于硬酸, 因而与氧离子、氟离 子等硬碱的亲合能力强, 稀土的氟化物、氧化物等纳米 材料更容易通过高温有机溶剂中的前驱体热分解法合 成得到. 然而, 稀土 +3 价离子与硫离子等软碱的亲合 能力弱, 因此稀土硫氧化物纳米颗粒的合成更加困难.

高松研究组首先以一种稀土的含硫配合物为前驱 体, 通过单源前驱体分解法在油胺中合成得到了 EuS 纳 米立方体 ${ }^{[12]}$ 和 $\mathrm{Eu}$ 和 $\mathrm{Gd}$ 的硫氧化物纳米板 ${ }^{[25]}$. 本组前期 工作首次通过高温有机溶剂中的前驱体热分解法合成 了钠离子掺杂的部分轻稀土和中稀土( $\mathrm{La}, \mathrm{Pr}, \mathrm{Nd}, \mathrm{Sm} \sim$ $\mathrm{Tb})$ 的硫氧化物 $\left(\mathrm{RE}_{2} \mathrm{O}_{2} \mathrm{~S}\right)$ 纳米板. 钠离子掺杂可以提高 六方相稀土硫氧化物的热力学稳定性 ${ }^{[23]}$. 提高钠离子 加入量, 也可以用类似方法合成得到 $\mathrm{NaRES}_{2}$ 型硫化物 复盐纳米材料 ${ }^{[24]}$. 但是用该方法仍无法合成其它中重 稀土( $\mathrm{Y}, \mathrm{Dy} \sim \mathrm{Lu}$ ) 硫氧化物纳米材料(由于 $\mathrm{Y}^{3+}$ 的离子半 径介于 $\mathrm{Ho}^{3+}$ 和 $\mathrm{Er}^{3+}$ 之间, 其软硬程度及与阴离子的亲 合能力与这两种镧系元素 +3 价阳离子相近, 因此, 本 文中将钎划在中重稀土元素范围内). 我们通过密度泛 函理论计算发现, 从轻稀土元素到重稀土元素, 其硫氧 化物的热力学稳定性降低, 得到对应氧化物的趋势增 大. 本文报道一种制备单分散中重稀土硫氧化物纳米板 的合成方法. 该方法是在纯油胺溶剂中使用分解温度较 稀土油酸盐更低的稀土乙酰丙酮盐为反应前驱体，以硫 化能力更强的硫化氢气体为硫化试剂, 在 $280{ }^{\circ} \mathrm{C}$ 下进 行反应. 该方法有效提高了反应体系中硫元素的化学 势, 弥补了三价中重稀土离子(除 $\mathrm{Lu}^{3+}$ 外)由于硬度高而 与硫离子弱的亲合作用, 因而利于它们的硫氧化物纳米 晶的生成. 用该方法可合成非掺杂的或者钠离子掺杂的 稀土硫氧化物纳米板. 其中, 所合成的 $\mathrm{Y}_{2} \mathrm{O}_{2} \mathrm{~S}: \mathrm{Eu}$ 纳米板 可在紫外光激发下发出红色荧光, 且纳米材料与体相材 料的苂光性质具有显著差别 ${ }^{[26 ~ 28]}$.

\section{2 结果与讨论}

\section{$2.1 \mathrm{RE}_{2} \mathrm{O}_{2} \mathrm{~S}$ 纳米板的结构表征}

在油胺与十八烯的混合溶剂中, 以稀土的乙酰丙酩 盐为前驱体, $280{ }^{\circ} \mathrm{C}$ 下加热分解, 以 $\mathrm{H}_{2} \mathrm{~S}$ 气体作为硫源, 可以合成得到 $\mathrm{Y}, \mathrm{Eu}, \mathrm{Gd}, \mathrm{Er}$ 和 $\mathrm{Yb}$ 的硫氧化物纳米板. 相应的透射电子显微镜(transmission electron microscopy, TEM)照片如图 $1 \mathrm{a}, \mathrm{c} \sim \mathrm{f}$ 所示. 这五种纳米板的平 均直径依次为 $(5.6 \pm 1.1) \mathrm{nm},(7.6 \pm 0.7) \mathrm{nm},(7.2 \pm 1.5)$ $\mathrm{nm},(12 \pm 2) \mathrm{nm},(14 \pm 5) \mathrm{nm} . \mathrm{Y}_{2} \mathrm{O}_{2} \mathrm{~S}$ 纳米板的选区电子 衍射(selected area electron diffraction, SAED)表征结果 和 $\mathrm{X}$ 射线能谱分析(Energy dispersive X-ray analysis, EDS)结果见支持信息. 通过 SAED 图案得出纳米材料 的晶面间距，可以与六方相 $\mathrm{Y}_{2} \mathrm{O}_{2} \mathrm{~S}$ 晶体晶面间距相吻 合. 且(100)和(110)衍射环最为明显, 说明 $\mathrm{Y}_{2} \mathrm{O}_{2} \mathrm{~S}$ 纳米板 在这两个方向上尺度较大, $\mathrm{Y}_{2} \mathrm{O}_{2} \mathrm{~S}$ 纳米板底面为(001)晶 面, 板的厚度方向为 $z$ 方向. EDS 表征结果表明 $\mathrm{Y}_{2} \mathrm{O}_{2} \mathrm{~S}$ 纳米板中 $\mathrm{Y}$ 和 $\mathrm{S}$ 的原子数比例为 $61: 39$, 基本吻合 2 : 1 的化学计量比. 图 2 为 $\mathrm{RE}_{2} \mathrm{O}_{2} \mathrm{~S}$ 纳米板的粉末 $\mathrm{X}$ 射线 衍射(X-ray diffraction, XRD)表征结果. 这些 XRD 谱图 均与相应的六方相 $\mathrm{RE}_{2} \mathrm{O}_{2} \mathrm{~S}$ 晶体粉末 $\mathrm{XRD}$ 标准谱图匹 配, 证明了合成得到的纳米板均为六方相 $\mathrm{RE}_{2} \mathrm{O}_{2} \mathrm{~S}$. 这 些 XRD 谱图中, $l$ 指标不为零的衍射峰宽化程度更高, 而且(003), (111)等衍射峰几乎观察不到, 说明这些硫氧 化物的纳米板在 $z$ 方向受限, 纳米板的底面为六方相 $\mathrm{RE}_{2} \mathrm{O}_{2} \mathrm{~S}$ 的(001)晶面. 衍射峰的宽化一方面源于纳米颗 粒尺寸较小, 原子排布的周期数较少; 另一方面源于纳 米颗粒中存在的缺陷导致的晶化度降低. 以(110)衍射 峰为例, 用硫氧化物纳米板的直径作为垂直于(110)晶 面方向的晶粒厚度, 可以通过 Scherrer 公式计算出纳米 板直径导致的(110)衍射峰的宽化程度. Scherrer 公式为:

$$
\beta_{110}=\frac{0.89 \lambda}{D_{110} \cos \theta}
$$

式中, $\beta_{110}$ 为纳米板直径导致的(110)衍射峰的半峰宽, 单位为弧度; $\lambda$ 为 $\mathrm{X}$ 射线波长, 此处为 $0.154 \mathrm{~nm} ; D_{110}$ 为 纳米板垂直于 (110) 晶面方向的晶粒厚度, 此处用纳米 板直径代替, 单位为 $\mathrm{nm} ; \theta$ 为衍射峰对应的 Bragg 角, 单 位为弧度. 使用该公式计算出 5 种稀土元素 $(Y, E u, G d$, Er 和 $\mathrm{Yb}$ )的硫氧化物纳米板由直径导致的(110)衍射峰 的半峰宽依次为 $1.5^{\circ}, 1.1^{\circ}, 1.2^{\circ}, 0.7^{\circ}$ 和 $0.6^{\circ}$. 用 PseudoViogt 函数对实测的(110)衍射峰进行拟合, 得到五种硫 氧化物纳米板该衍射峰的半峰宽依次为 $2.2^{\circ}, 1.8^{\circ}, 1.6^{\circ}$, $2.8^{\circ}$ 和 $1.0^{\circ}$. 可见, 对于 $\mathrm{Gd}_{2} \mathrm{O}_{2} \mathrm{~S}$ 和 $\mathrm{Yb}_{2} \mathrm{O}_{2} \mathrm{~S}$ 纳米板, 晶化 度导致的衍射峰展宽较少, 而对于 $\mathrm{Er}_{2} \mathrm{O}_{2} \mathrm{~S}$ 纳米板, 衍射 峰展宽主要源于低的晶化度.

本组之前工作已经证明 $\mathrm{Na}^{+}$离子掺杂可以提高六方 相 $\mathrm{La}_{2} \mathrm{O}_{2} \mathrm{~S}$ 晶体的稳定性, 利于 $\mathrm{La}_{2} \mathrm{O}_{2} \mathrm{~S}: \mathrm{Na}$ 纳米板的形 成 ${ }^{[23]}$. 在本工作使用的合成方法中, 通过引入油酸钠进 行 $\mathrm{Na}^{+}$离子掺杂, 同样可以稳定 $\mathrm{Y}_{2} \mathrm{O}_{2} \mathrm{~S}$ 晶格, 使纳米板 的晶化程度提高. 图 $1 \mathrm{~b}$ 为合成得到的 $\mathrm{Y}_{2} \mathrm{O}_{2} \mathrm{~S}: \mathrm{Na}$ 纳米板 的 TEM 照片, 纳米板的平均直径为 $(8.4 \pm 0.7) \mathrm{nm}$. 相应 的 EDS 表征结果见支持信息, 其中 $\mathrm{Y}$ 和 $\mathrm{Na}$ 的原子个数 比例为 $71: 29 . \mathrm{Y}_{2} \mathrm{O}_{2} \mathrm{~S}: \mathrm{Na}$ 纳米板的 $\mathrm{XRD}$ 表征结果见图 2 , 该谱图可以与六方相 $\mathrm{Y}_{2} \mathrm{O}_{2} \mathrm{~S}$ 晶体标准谱图匹配. 根 据 Sherrer 公式计算出 $\mathrm{Y}_{2} \mathrm{O}_{2} \mathrm{~S}: \mathrm{Na}$ 纳米板由纳米板尺寸导 致的 (110)衍射峰的半峰宽为 $1.0^{\circ}\left(\mathrm{Y}_{2} \mathrm{O}_{2} \mathrm{~S}\right.$ 纳米板为 $\left.1.5^{\circ}\right)$, 用 Pseudo-Viogt 函数对实测的(110)衍射峰拟合得到该 衍射峰的半峰宽为 $1.3^{\circ}\left(\mathrm{Y}_{2} \mathrm{O}_{2} \mathrm{~S}\right.$ 纳米板为 $\left.2.2^{\circ}\right)$. 可见，与 

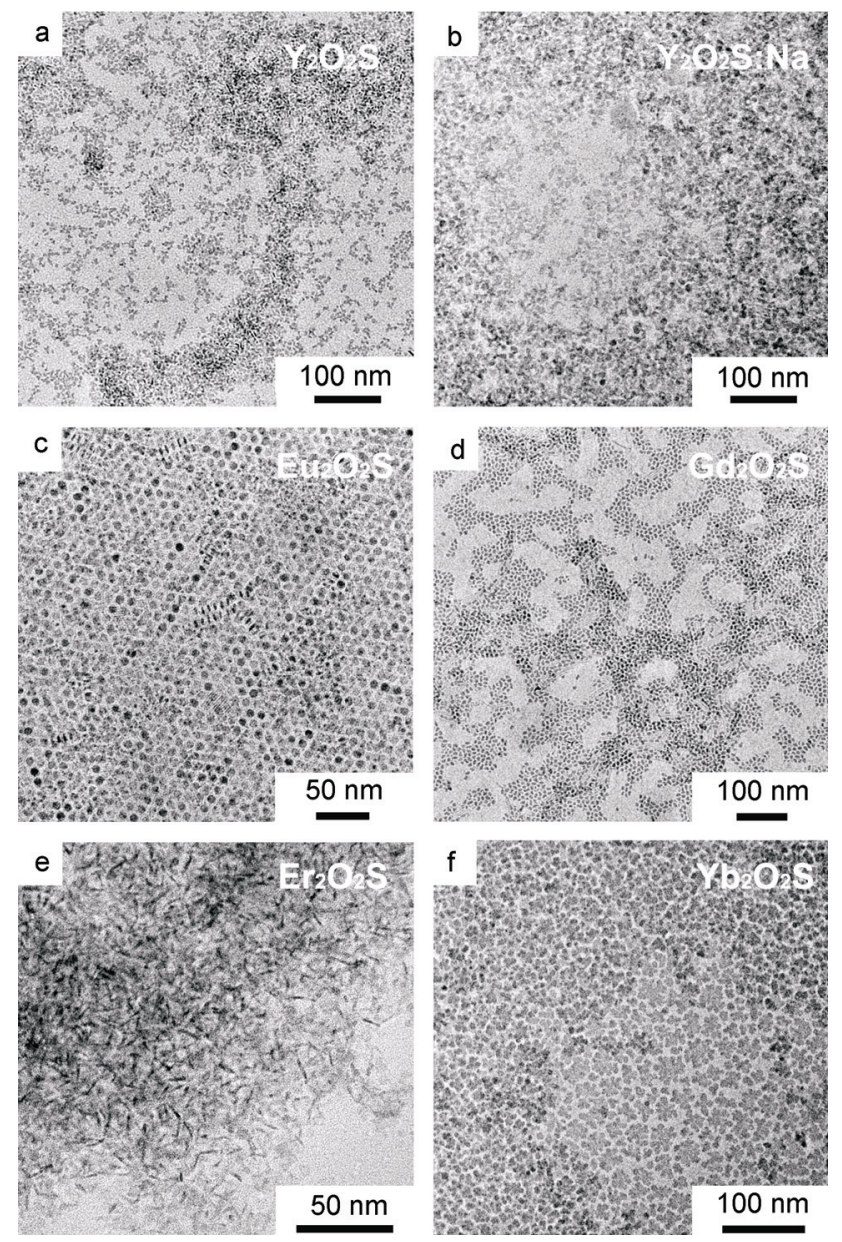

图 $1 \mathrm{RE}_{2} \mathrm{O}_{2} \mathrm{~S}$ 及 $\mathrm{Y}_{2} \mathrm{O}_{2} \mathrm{~S}: \mathrm{Na}$ 纳米板的 TEM 照片

Figure 1 TEM images of $\mathrm{RE}_{2} \mathrm{O}_{2} \mathrm{~S}$ and $\mathrm{Y}_{2} \mathrm{O}_{2} \mathrm{~S}$ :Na nanoplates

无 $\mathrm{Na}^{+}$离子掺杂的 $\mathrm{Y}_{2} \mathrm{O}_{2} \mathrm{~S}$ 纳米板相比, $\mathrm{Y}_{2} \mathrm{O}_{2} \mathrm{~S}: \mathrm{Na}$ 纳米板 由晶化程度造成的衍射峰宽化程度较低, 说明 $\mathrm{Na}^{+}{ }^{+}$离子 的引入可以提高纳米板的晶化程度.

\section{$2.2 \mathrm{Y}_{2} \mathrm{O}_{2} \mathrm{~S}: \mathrm{Na}$ 纳米板的形成过程}

图 3 为合成 $\mathrm{Y}_{2} \mathrm{O}_{2} \mathrm{~S}$ 纳米板的前驱体乙酰丙酮钎在氮 气中的热重分析(thermogravimetric analysis, TGA)曲线. 乙酰丙酮钎在氮气中加热完全分解的固态产物为 $\mathrm{Y}_{2} \mathrm{O}_{3}$. 根据图 3 中的失重曲线, 可以计算得到在 $200{ }^{\circ} \mathrm{C}$ 时前驱 体的存在形式是无水乙酰丙酮钇. 低于 $200{ }^{\circ} \mathrm{C}$ 阶段的 失重来源于前驱体失去结晶水, 据此得到实验中使用的 乙酰丙酮钎的组成为 $\mathrm{Y}\left(\mathrm{C}_{5} \mathrm{H}_{7} \mathrm{O}_{2}\right)_{3} \cdot 2.7 \mathrm{H}_{2} \mathrm{O}$. 该前驱体的 主要失重温度区间为 $200 \sim 300{ }^{\circ} \mathrm{C}$, 其中在 $250{ }^{\circ} \mathrm{C}$ 附近 前驱体失重最多. 热重分析表明, 乙酰丙酮钎作为合成 反应的前驱体, 其分解主要发生在 $200 \sim 300{ }^{\circ} \mathrm{C}$ 的温度 范围内, 该温度低于稀土油酸盐的热分解温度 ${ }^{[29]}$.

图 4 为在合成 $\mathrm{Y}_{2} \mathrm{O}_{2} \mathrm{~S}: \mathrm{Na}$ 纳米板的过程中, 随反应 温度升高反应体系中产生的纳米颗粒的 TEM 照片. 当 反应体系温度升高到 $220{ }^{\circ} \mathrm{C}$ 时，乙酰丙酮钎前驱体已 经开始分解，并有超薄纳米板产生，直径为(15 \pm 2$) \mathrm{nm}$, 部分纳米板通过面面堆积自组装形成一维超晶格结构.

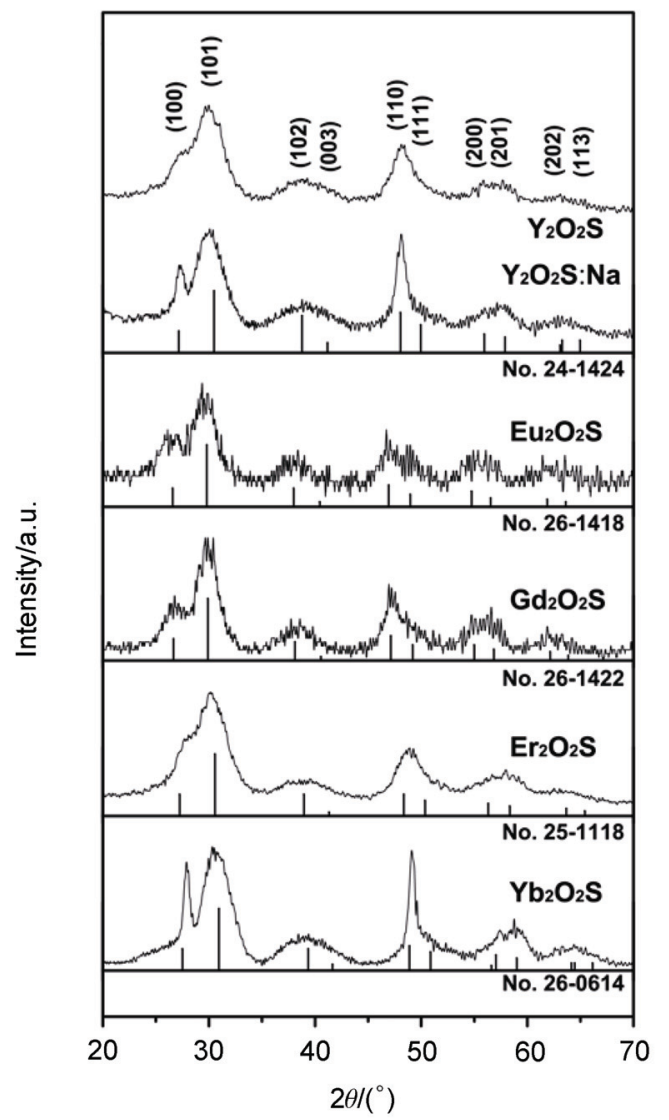

图 $2 \mathrm{RE}_{2} \mathrm{O}_{2} \mathrm{~S}$ 及 $\mathrm{Y}_{2} \mathrm{O}_{2} \mathrm{~S}: \mathrm{Na}$ 纳米板的 XRD 谱图

Figure 2 XRD patterns of $\mathrm{RE}_{2} \mathrm{O}_{2} \mathrm{~S}$ and $\mathrm{Y}_{2} \mathrm{O}_{2} \mathrm{~S}$ :Na nanoplates

The XRD patterns are compared with the standard diffraction peaks of corresponding $\mathrm{RE}_{2} \mathrm{O}_{2} \mathrm{~S}$ crystals without Na dopant. The corresponding JCPDS card numbers are also listed.

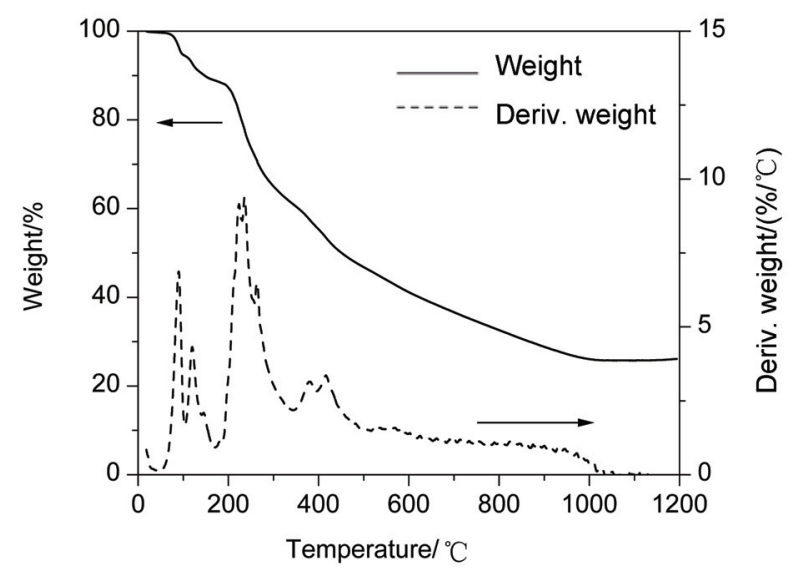

图 3 乙酰丙酮钎在氮气气氛下的热重曲线

Figure 3 TGA analysis of $\mathrm{Y}(\mathrm{acac})_{3}$ in $\mathrm{N}_{2}$

The solid line stands for the weight of the sample and the dashed line stands for the derivative of the weight with respect to the temperature

$260 \sim 270{ }^{\circ} \mathrm{C}$ 这些纳米板逐渐溶解碎裂, 在 $270{ }^{\circ} \mathrm{C}$ 时纳 米板的直径为 $(8 \pm 1) \mathrm{nm}$. 此后纳米板的尺寸不再发生 明显变化, 在 $280{ }^{\circ} \mathrm{C}$ 反应 $1 \mathrm{~h}$ 后得到的 $\mathrm{Y}_{2} \mathrm{O}_{2} \mathrm{~S}: \mathrm{Na}$ 纳米 板的直径为 $(8.4 \pm 0.7) \mathrm{nm}$. 


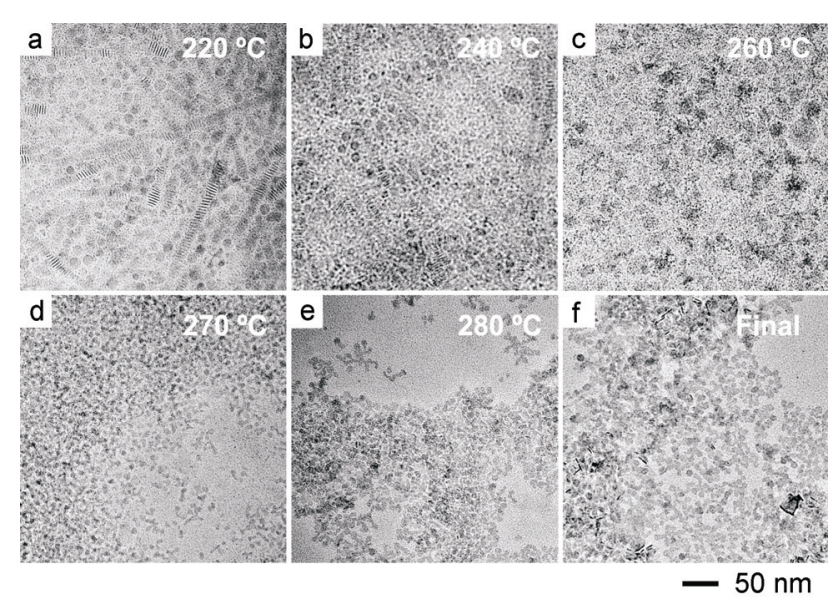

图 4 在合成 $\mathrm{Y}_{2} \mathrm{O}_{2} \mathrm{~S}: \mathrm{Na}$ 纳米板的升温过程中所获得的纳米颗粒的 TEM 照片

Figure 4 TEM images of the as-obtained nanoparticles at different temperatures during the heating process in the synthesis of $\mathrm{Y}_{2} \mathrm{O}_{2} \mathrm{~S}: \mathrm{Na}$ nanoplates.

(a) $220{ }^{\circ} \mathrm{C}$; (b) $240{ }^{\circ} \mathrm{C}$; (c) $260{ }^{\circ} \mathrm{C}$; (d) $270{ }^{\circ} \mathrm{C}$; (e) $280{ }^{\circ} \mathrm{C}$; (f) final product kept at $280{ }^{\circ} \mathrm{C}$ for $1 \mathrm{~h}$

对不同温度下所获得的纳米板进行 EDS 表征, 计 算每份样品中 $\mathrm{S}, \mathrm{Y}$ 和 $\mathrm{Na}$ 的元素比例, 结果如图 5 所示. 当反应温度为 $220{ }^{\circ} \mathrm{C}$ 时, 三种元素摩尔比为 $\mathrm{S}: \mathrm{Y}: \mathrm{Na}$ $=3: 67: 30$, 纳米板中硫元素含量很低, 表明该纳米 板为 $\mathrm{Y}_{2} \mathrm{O}_{3}: \mathrm{Na}$ 纳米板. 当温度超过 $240{ }^{\circ} \mathrm{C}$ 后, 纳米板中 的硫元素含量逐渐升高. 当温度升高到 $280{ }^{\circ} \mathrm{C}$ 时, 三种 元素的摩尔比为 $\mathrm{S}: \mathrm{Y}: \mathrm{Na}=19: 62: 19$; 反应溶液保 持在 $280{ }^{\circ} \mathrm{C} 1 \mathrm{~h}$ 之后, 三种元素摩尔比为 $\mathrm{S}: \mathrm{Y}: \mathrm{Na}=$ $23: 53: 24$. 可见, 随着反应温度的升高和反应时间的 延长, 纳米板中的硫元素含量逐渐升高.

结合 TGA, TEM 和 EDS 表征结果可知, 在 $\mathrm{Y}_{2} \mathrm{O}_{2} \mathrm{~S}$ : $\mathrm{Na}$ 纳米板的合成过程中, 在 $220{ }^{\circ} \mathrm{C}$ 时, 前驱体乙酰丙 酮钎已经开始分解, 首先产生的是 $\mathrm{Y}_{2} \mathrm{O}_{3}: \mathrm{Na}$ 纳米板, 在 有 $\mathrm{H}_{2} \mathrm{~S}$ 气体存在的气氛下, 当温度超过 $240{ }^{\circ} \mathrm{C}$ 时, $\mathrm{Y}_{2} \mathrm{O}_{3}$ : $\mathrm{Na}$ 纳米板逐渐破碎并被逐步硫化, 最终生成 $\mathrm{Y}_{2} \mathrm{O}_{2} \mathrm{~S}: \mathrm{Na}$ 纳米板. 产品中较高的钠元素含量可能来自于纳米板表 面吸附的油酸钠.

\section{3 不同稀土元素形成硫氧化物纳米板的趋势}

镧的硫氧化物纳米板可以通过钠离子掺杂在含有 油酸的混合溶剂中以硫单质为硫源合成得到 ${ }^{[23]}$; 钎的 硫氧化物纳米板可以通过使用不含油酸的混合溶剂, 以 $\mathrm{H}_{2} \mathrm{~S}$ 气体为硫源合成得到; 而使用以上两种合成方法, 都无法合成得到镥的硫氧化物纳米材料. 按照合成 $\mathrm{Y}_{2} \mathrm{O}_{2} \mathrm{~S}$ 纳米板的合成方法, 将前驱体乙酰丙酮钎替换成 等物质量的乙酰丙酮镥, 得到的产物为 $\mathrm{Lu}_{2} \mathrm{O}_{3}$ 纳米颗粒, 相应的 TEM 和 XRD 表征结果见支持信息. 这些实验结 果表明, 从轻稀土元素过渡到重稀土元素, 合成硫氧化 物纳米板的难度逐渐增大, 得到氧化物纳米板的可能性 增大.

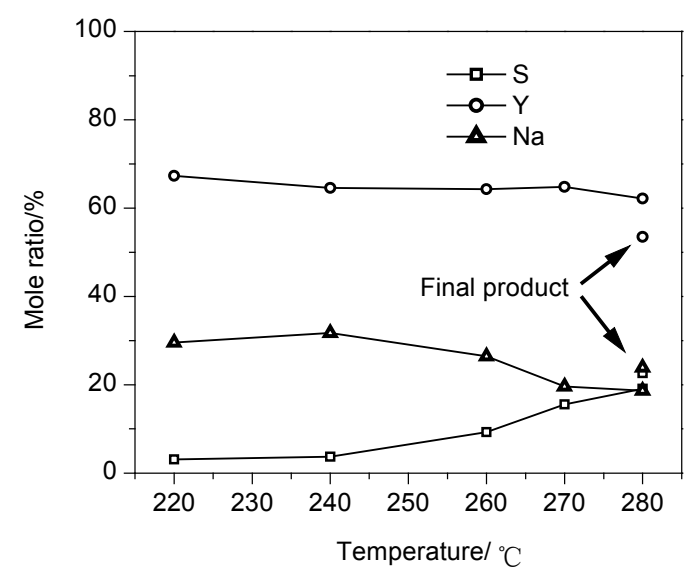

图 $5 \mathrm{EDS}$ 表征给出的升温过程中不同温度下的纳米板及最终产物中 $\mathrm{S}, \mathrm{Y}$ 和 $\mathrm{Na}$ 元素的摩尔比例

Figure $5 \mathrm{~S}: \mathrm{Y}:$ Na mole ratio of the nanoplates taken out at different temperatures during the heating up period and the final product obtained from EDS analysis

本工作通过密度泛函理论(density functional theory， DFT)计算，模拟了镧、钎、镥的氧化物和硫氧化物的相 对热力学稳定性, 并对以上实验现象做出了解释. 考虑 下式描述的化学转化过程:

$\mathrm{RE}_{2} \mathrm{O}_{3}+\mathrm{S} \leftrightarrow \mathrm{RE}_{2} \mathrm{O}_{2} \mathrm{~S}+\mathrm{O}$

该过程的摩尔自由能变为:

$\Delta G_{\mathrm{m}}=\left[\Delta_{\mathrm{f}} G_{\mathrm{m}}\left(\mathrm{RE}_{2} \mathrm{O}_{2} \mathrm{~S}\right)-\Delta_{\mathrm{f}} G_{\mathrm{m}}\left(\mathrm{RE}_{2} \mathrm{O}_{3}\right)\right]-\left(\mu_{\mathrm{S}}-\mu_{\mathrm{O}}\right)(3)$ 式(3)中, $\Delta_{\mathrm{f}} G_{\mathrm{m}}\left(\mathrm{RE}_{2} \mathrm{O}_{2} \mathrm{~S}\right)$ 和 $\Delta_{\mathrm{f}} G_{\mathrm{m}}\left(\mathrm{RE}_{2} \mathrm{O}_{3}\right)$ 分别为 $\mathrm{RE}_{2} \mathrm{O}_{2} \mathrm{~S}$ 和 $\mathrm{RE}_{2} \mathrm{O}_{3}$ 的摩尔生成自由能; $\mu_{\mathrm{S}}$ 和 $\mu_{\mathrm{O}}$ 分别为硫元素和氧元 素在合成环境中的化学势. 要求式(2)中所示的过程向 右是自发的, 即 $\mathrm{RE}_{2} \mathrm{O}_{2} \mathrm{~S}$ 是热力学稳定的, 则要求式(3) 中 $\Delta G_{\mathrm{m}}<0$, 即:

$$
\mu_{\mathrm{S}}-\mu_{\mathrm{O}}>\Delta_{\mathrm{f}} G_{\mathrm{m}}\left(\mathrm{RE}_{2} \mathrm{O}_{2} \mathrm{~S}\right)-\Delta_{\mathrm{f}} G_{\mathrm{m}}\left(\mathrm{RE}_{2} \mathrm{O}_{3}\right)
$$

通过 DFT 计算可以得到 $\mathrm{La}, \mathrm{Y}$ 和 $\mathrm{Lu}$ 三种稀土元素 的 $\Delta_{\mathrm{f}} G_{\mathrm{m}}\left(\mathrm{RE}_{2} \mathrm{O}_{2} \mathrm{~S}\right)$ 和 $\Delta_{\mathrm{f}} G_{\mathrm{m}}\left(\mathrm{RE}_{2} \mathrm{O}_{3}\right)$ 的值, 进而计算 $\left[\Delta_{\mathrm{f}} G_{\mathrm{m}}\left(\mathrm{RE}_{2} \mathrm{O}_{2} \mathrm{~S}\right)-\Delta_{\mathrm{f}} G_{\mathrm{m}}\left(\mathrm{RE}_{2} \mathrm{O}_{3}\right)\right]$. 从而得到对于这三种元 素在热力学稳定条件下获得硫氧化物 $\left(\mu_{\mathrm{S}}-\mu_{\mathrm{O}}\right)$ 可取的最 小值, 计算结果如表 1 所示. $\left(\mu_{\mathrm{S}}-\mu_{\mathrm{O}}\right)$ 可取值越低, 硫氧 化物就可以在氧化学势越高, 硫化学势越低的环境中稳 定存在而不会转化为氧化物, 即硫氧化物的热力学稳定 性越高. 分析该表格中的数据可以发现, 钠掺杂的铗体 系具有最低的 $\left(\mu_{\mathrm{S}}-\mu_{\mathrm{O}}\right)$ 取值下限, 钎体系的 $\left(\mu_{\mathrm{S}}-\mu_{\mathrm{O}}\right)$ 取值 下限较针体系高 $0.6 \mathrm{eV}$, 镥体系较钇体系高 $0.2 \mathrm{eV}$. 所 以说, 钠离子掺杂可以提高 $\mathrm{La}_{2} \mathrm{O}_{2} \mathrm{~S}$ 的热力学稳定性, $\mathrm{Y}_{2} \mathrm{O}_{2} \mathrm{~S}$ 的热力学稳定性较 $\mathrm{La}_{2} \mathrm{O}_{2} \mathrm{~S}$ 差, $\mathrm{Lu}_{2} \mathrm{O}_{2} \mathrm{~S}$ 热力学稳定 性最差. 值得一提的是, 在以上计算中我们忽略了表界 面效应对于纳米颗粒总生成能的贡献，但由于我们是在 比较氧化物和硫氧化物两种纳米颗粒的相对稳定性，因 此这种贡献在比较中会被抵消，所以通过以上计算过程 比较硫氧化物和氧化物纳米颗粒的相对稳定性是合理 的. 
表 $1 \mathrm{La}, \mathrm{Y}, \mathrm{Lu}$ 三种稀土元素 $\left[\Delta_{\mathrm{f}} G_{\mathrm{m}}\left(\mathrm{RE}_{2} \mathrm{O}_{2} \mathrm{~S}\right)-\Delta_{\mathrm{f}} G_{\mathrm{m}}\left(\mathrm{RE}_{2} \mathrm{O}_{3}\right)\right]$ 值的 DFT 计算结果

Table 1 The value of $\left[\Delta_{\mathrm{f}} G_{\mathrm{m}}\left(\mathrm{RE}_{2} \mathrm{O}_{2} \mathrm{~S}\right)-\Delta_{\mathrm{f}} G_{\mathrm{m}}\left(\mathrm{RE}_{2} \mathrm{O}_{3}\right)\right]$ for $\mathrm{La}, \mathrm{Y}$ and $\mathrm{Lu}$ obtained through DFT calculations

\begin{tabular}{lccc}
\hline $\mathrm{RE}$ & $\begin{array}{c}\Delta_{\mathrm{f}} G_{\mathrm{m}}\left(\mathrm{RE}_{2} \mathrm{O}_{2} \mathrm{~S}\right) \\
/ \mathrm{eV}\end{array}$ & $\begin{array}{c}\Delta_{\mathrm{f}} G_{\mathrm{m}}\left(\mathrm{RE}_{2} \mathrm{O}_{3}\right) \\
/ \mathrm{eV}\end{array}$ & $\begin{array}{c}\Delta_{\mathrm{f}} G_{\mathrm{m}}\left(\mathrm{RE}_{2} \mathrm{O}_{2} \mathrm{~S}\right)-\Delta_{\mathrm{f}} G_{\mathrm{m}}\left(\mathrm{RE}_{2} \mathrm{O}_{3}\right) \\
/ \mathrm{eV}\end{array}$ \\
\hline $\mathrm{La}^{a}$ & -16.1 & -17.4 & 1.3 \\
$\mathrm{La}^{\mathrm{Na}}{ }^{a, b}$ & & & 1.1 \\
$\mathrm{Y}$ & -15.9 & -17.8 & 1.9 \\
$\mathrm{Lu}$ & -15.7 & -17.8 & 2.1 \\
\hline
\end{tabular}

${ }^{a}$ These data are extracted from ref. ${ }^{[23]} \cdot{ }^{b} \mathrm{La}: \mathrm{Na}$ stands for sodium doped $\mathrm{La}_{2} \mathrm{O}_{2} \mathrm{~S}$ and $\mathrm{La}_{2} \mathrm{O}_{3}$. The data mean the lower limit of $\left(\mu_{\mathrm{S}}-\mu_{\mathrm{O}}\right)$ to thermodynamically stabilized $\mathrm{La}_{2} \mathrm{O}_{2} \mathrm{~S}: \mathrm{Na}$ is $1.1 \mathrm{eV}$.

相比于 $\mathrm{La}_{2} \mathrm{O}_{2} \mathrm{~S}: \mathrm{Na}, \mathrm{Y}_{2} \mathrm{O}_{2} \mathrm{~S}$ 可稳定存在时要求的 $\left(\mu_{\mathrm{S}}-\mu_{\mathrm{O}}\right)$ 取值下限较高, 因此, 合成 $\mathrm{Y}_{2} \mathrm{O}_{2} \mathrm{~S}$ 纳米板时, 需 要提高硫元素的化学势同时降低氧元素的化学势. 在合 成反应开始前, 反应混合物需在 $100{ }^{\circ} \mathrm{C}$ 真空条件下除 水除氧. 该操作可以在一定程度上降低反应体系中氧元 素的化学势. 在本组之前工作中, $\mathrm{La}_{2} \mathrm{O}_{2} \mathrm{~S}: \mathrm{Na}$ 纳米板可以 在油酸-油胺一十八烯的混合溶剂中合成得到, 但在该混 合溶剂中无法合成 $\mathrm{Y}_{2} \mathrm{O}_{2} \mathrm{~S}$ 或 $\mathrm{Y}_{2} \mathrm{O}_{2} \mathrm{~S}: \mathrm{Na}$ 纳米板. 该溶剂 体系酸度较高, 硫化氢的溶解度小, 且稀土乙酰丙酮盐 会先与油酸反应生成稀土油酸盐. 稀土油酸盐分解温度 高于 $300{ }^{\circ} \mathrm{C}$, 而高温不利于硫化氢的溶解. 因此, 该体 系中硫化氢浓度较低, 硫元素的化学势较低, 无法生成 $\mathrm{Y}_{2} \mathrm{O}_{2} \mathrm{~S}: \mathrm{Na}$ 纳米板. 而在本工作中, 仅用油胺做溶剂, 不 加入油酸, 以提高硫化氢的溶解度; 以稀土乙酰丙酮盐 为前驱体, 利用稀土乙酰丙酮盐较低的分解温度, 在低 于 $300{ }^{\circ} \mathrm{C}$ 的条件下合成 $\mathrm{Na}^{+}$离子掺杂的或非掺杂的 $\mathrm{Y}_{2} \mathrm{O}_{2} \mathrm{~S}$ 纳米板, 同样利于硫化氢溶解, 提高硫元素的化 学势. 此外, 合成 $\mathrm{La}_{2} \mathrm{O}_{2} \mathrm{~S}: \mathrm{Na}$ 纳米板时, 硫化试剂为硫 单质，而本工作中使用了硫化氢气体. Ozin 等 ${ }^{[30]}$ 的工作 表明硫单质作为油胺溶液中的硫化试剂, 其原理为硫单 质首先被油胺还原为 -2 价, 而真正起到硫化作用的硫 物种硫的价态为 -2 价. 而直接使用硫化氢作为硫化试 剂, 在碱性的油胺溶液中更容易电离产生硫离子, 同样 起到了提高硫元素化学势, 增强硫化能力的目的. 然而, 要使 $\mathrm{Lu}_{2} \mathrm{O}_{2} \mathrm{~S}$ 稳定存在, 则需要更高的硫元素化学势. 使 用本工作中的合成方法, 仍无法达到如此高的硫元素的 化学势, 因而该方法只能得到 $\mathrm{Lu}_{2} \mathrm{O}_{3}$ 纳米板. 可见, 以 上 DFT 计算可以合理解释不同稀土元素形成硫氧化物 纳米板的趋势.

我们还可以从软硬酸碱理论的角度理解不同稀土 元素形成硫氧化物纳米板的趋势 ${ }^{[31]}$. 镧系元素从镧到 镥, $4 \mathrm{f}$ 电子逐渐增多; 由于 $4 \mathrm{f}$ 电子云位于内层, 但对于 核电荷的屏蔽较弱, 因此对于 +3 价离子而言, 外层 $5 \mathrm{~s}$ 和 $5 \mathrm{p}$ 电子受到的有效核电荷吸引逐渐变弱, 其离子半 径逐渐减小. $\mathrm{Y}^{3+}$ 离子相比于 $\mathrm{La}^{3+}$ 离子, 少一层电子, 因 而其离子半径比 $\mathrm{La}^{3+}$ 离子的小. $\mathrm{Y}^{3+}$ 离子的半径与 $\mathrm{Er}^{3+}$ 离子的接近. 因此, 离子半径比较: $\mathrm{La}^{3+}>\mathrm{Y}^{3+}>\mathrm{Lu}^{3+}$.
由软硬酸碱理论可知，离子所带电荷越高，离子半径越 小, 离子的硬度越高. +3 价稀土元素离子由于具有高 的电荷数, 都属于硬酸; 而硫离子的半径比氧离子的大, 因此硫离子属于软碱. 硬酸与软碱亲合能力弱. 相比而 言, 轻稀土元素离子半径较大, 硬度较低, 与硫离子亲 合能力稍强, 中稀土和重稀土元素离子半径较小, 硬度 更高, 与硫离子亲和能力更弱. 所以, 轻稀土元素的硫 氧化物纳米板更容易合成得到, 而重稀土元素更倾向于 形成氧化物. 对于离子半径最小的 $\mathrm{Lu}^{3+}$ 离子, 本工作中 的合成方法只能得到 $\mathrm{Lu}_{2} \mathrm{O}_{3}$ 纳米板.

\section{$2.4 \mathrm{Y}_{2} \mathrm{O}_{2} \mathrm{~S}: \mathrm{Eu}$ 纳米板的荧光性质}

稀土硫氧化物是一类重要的无机苂光材料, 其中, $\mathrm{Y}_{2} \mathrm{O}_{2} \mathrm{~S}: \mathrm{Eu}$ 是一种被广泛应用的红色荧光材料. 图 6 为 $\mathrm{Y}_{2} \mathrm{O}_{2} \mathrm{~S}: \mathrm{Eu}$ 纳米板环已烷分散液与其体相材料的荧光光 谱对比(纳米板的直径为 $6.1 \pm 1.1 \mathrm{~nm}$, 相应的 TEM 照片 见支持信息). 其中, EDS 表征表明 $\mathrm{Y}_{2} \mathrm{O}_{2} \mathrm{~S}: \mathrm{Eu}$ 纳米板中 $\mathrm{Eu}$ 的掺杂比例为 $4.5 \%$ (EDS 谱图见支持信息), 体相材 料 $\mathrm{Eu}$ 的掺杂量为 $4 \%$. 测试 $\mathrm{Y}_{2} \mathrm{O}_{2} \mathrm{~S}: \mathrm{Eu}$ 的体相和纳米相 荧光激发光谱使用的出射波长均为最大荧光强度的对 应波长, 即 $625 \mathrm{~nm}$. 测定 $\mathrm{Y}_{2} \mathrm{O}_{2} \mathrm{~S}: \mathrm{Eu}$ 纳米板环己烷分散 液的发射光谱使用的激发波长为其获得最大荧光强度 对应的激发波长, 即 $251 \mathrm{~nm}$; 同理, 测定 $\mathrm{Y}_{2} \mathrm{O}_{2} \mathrm{~S}$ : Eu 体相 材料发射光谱使用的激发波长为 $350 \mathrm{~nm}$. $\mathrm{Eu}^{3+}$ 离子掺杂 在 $\mathrm{Y}_{2} \mathrm{O}_{2} \mathrm{~S}$ 晶格中产生的红色荧光发射主要源于 $\mathrm{Eu}^{3+}$ 离 子的 ${ }^{5} \mathrm{D}_{0} \rightarrow{ }^{7} \mathrm{~F}_{J}(J=0 \sim 2,4)$ 跃迁 ${ }^{[26,28]}$. $\mathrm{Y}_{2} \mathrm{O}_{2} \mathrm{~S}$ : $\mathrm{Eu}$ 纳米板中 的 $\mathrm{Eu}^{3+}$ 离子所有发射峰均有所宽化, 主要源于纳米相 材料中较高的缺陷浓度和表面效应. $\mathrm{Y}_{2} \mathrm{O}_{2} \mathrm{~S}$ : $\mathrm{Eu}$ 纳米板与 其体相材料发出荧光的色坐标(CIE 1931)分别为 (0.64, 0.35 )和 $(0.66,0.34)$ (色坐标图见支持信息). 而 $\mathrm{Y}_{2} \mathrm{O}_{2} \mathrm{~S}: \mathrm{Eu}$ 纳米板与其体相材料的激发光谱有着显著差别: 纳米板

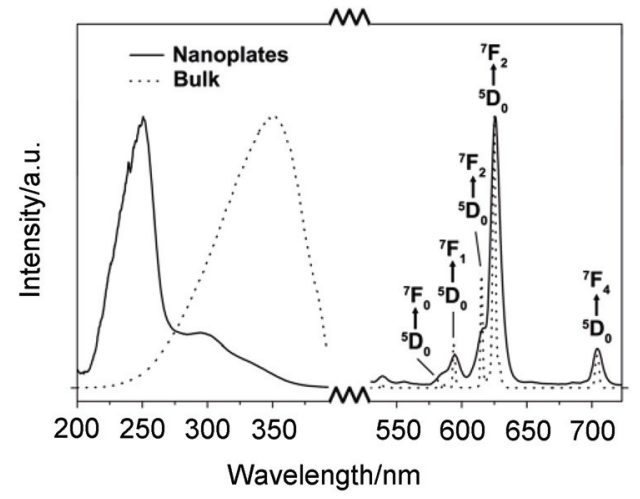

图 $6 \mathrm{Y}_{2} \mathrm{O}_{2} \mathrm{~S}: \mathrm{Eu}$ 纳米板环已烷分散液与体相材料的荧光光谱

Figure 6 Fluorescence spectra of the as-synthesized $\mathrm{Y}_{2} \mathrm{O}_{2} \mathrm{~S}: \mathrm{Eu}$ nanoplates and the corresponding bulk counterpart

The left part is the excitation spectrum and the right part is the emission spectrum. The solid lines stand for the spectrum of the nanoplates and the dashed lines stand for that of the bulk counterpart. Both of the excitation spectra of these two samples were obtained at the emission wavelength of $625 \mathrm{~nm}$. The emission spectrum of $\mathrm{Y}_{2} \mathrm{O}_{2} \mathrm{~S}: \mathrm{Eu}$ nanoplates was obtained at the excitation wavelength of $251 \mathrm{~nm}$ and that of the bulky sample was obtained at the excitation wavelength of $350 \mathrm{~nm}$ 
的激发光谱相比体相材料具有明显蓝移, 且在 $250 \mathrm{~nm}$ 附近和 $300 \mathrm{~nm}$ 以上存在多个激发峰. 类似的情况在宋 宏伟等合成的 $\mathrm{La}_{2} \mathrm{O}_{2} \mathrm{~S}: \mathrm{Eu}$ 纳米颗粒 ${ }^{[27]}$ 及本组合成的钠掺 杂的 $\mathrm{La}_{2} \mathrm{O}_{2} \mathrm{~S}: \mathrm{Eu}$ 纳米板 ${ }^{[23]}$ 中均被观察到. 宋宏伟等通过 研究燃烧法合成得到的 $\mathrm{La}_{2} \mathrm{O}_{2} \mathrm{~S}: \mathrm{Eu}$ 纳米颗粒的荧光性 质, 发现其位于 $250 \mathrm{~nm}$ 和 $330 \mathrm{~nm}$ 附近的两个主要的激 发谱带分别源于 $\mathrm{Eu}-\mathrm{O}$ 和 $\mathrm{Eu}-\mathrm{S}$ 的电荷转移跃迁 ${ }^{[27]}$. 而在 本实验合成的 $\mathrm{Y}_{2} \mathrm{O}_{2} \mathrm{~S}$ : Eu 纳米板位于 $250 \mathrm{~nm}$ 附近的激发 峰明显强于 $300 \mathrm{~nm}$ 附近的激发峰, 表明其荧光的主要 激发过程是 $\mathrm{O}^{2-}$ 离子到 $\mathrm{Eu}^{3+}$ 离子的电荷转移, 这可能是 由于纳米板的表面稀土离子处在富氧的环境中, 这与本 组之前合成得到的钠掺杂的 $\mathrm{La}_{2} \mathrm{O}_{2} \mathrm{~S}: \mathrm{Eu}$ 纳米板类似 ${ }^{[23]}$.

$\mathrm{Y}_{2} \mathrm{O}_{2} \mathrm{~S}: \mathrm{Eu}$ 纳米板和体相材料在室温下的荧光衰减 曲线见支持信息. 测试两份样品分别使用了各自获得最 大苂光强度对应的激发波长, 发射波长均为 $625 \mathrm{~nm}$. 按 照公式(4)对曲线进行拟合:

$$
I=I_{0} e^{-t / \tau}
$$

拟合得到的纳米板和体相材料的苂光寿命 $\tau$ 分别为 $12 \mu \mathrm{s}$ 和 $518 \mu \mathrm{s}$. 此外, 本工作还测定了 $\mathrm{Y}_{2} \mathrm{O}_{2} \mathrm{~S}: \mathrm{Eu}$ 纳米 板和体相材料在波长为 $350 \mathrm{~nm}$ 的光激发下的菼光量子 产率(photoluminescence quantum yield, PLQY). $\mathrm{Y}_{2} \mathrm{O}_{2} \mathrm{~S}$ : $\mathrm{Eu}$ 纳米板的 PLQY 为 $1.49 \%$, 低于其体相材料的 PLQY $(35.06 \%) . \mathrm{Y}_{2} \mathrm{O}_{2} \mathrm{~S}: \mathrm{Eu}$ 纳米板较短的荧光寿命和较低的 PLQY 可能源于 $\mathrm{Y}_{2} \mathrm{O}_{2} \mathrm{~S}$ : Eu 纳米板的晶化程度较低. 纳米 板的晶格弛豫造成的非辐射越强使其发光量子产率显 著下降; 而且由于 $\mathrm{Y}_{2} \mathrm{O}_{2} \mathrm{~S}: \mathrm{Eu}$ 纳米板直径小、厚度薄, 表 面原子占整个颗粒原子比例很高, 因此纳米板的内部发 光中心吸收的能量很快传递到纳米板表面发光中心并 通过非辐射跃迁途径耗散掉 ${ }^{[32]}$.

\section{3 结论}

本工作通过油胺中稀土乙酰丙酮盐的热分解, 以硫 化氢气体为硫化试剂, 合成得到了除硫氧化镥外的中重 稀土硫氧化物纳米板. 以 $\mathrm{Y}_{2} \mathrm{O}_{2} \mathrm{~S}: \mathrm{Na}$ 纳米板为例, 前驱 体热重分析及合成过程中间产物的元素分析结果表明, 稀土乙酰丙酮盐在 $220{ }^{\circ} \mathrm{C}$ 已经分解, 首先产生的是稀 土氧化物纳米板, 在硫化氢饱和的油胺溶液中, 随着温 度升高, 纳米板中硫元素的比例逐渐升高, 在 $280{ }^{\circ} \mathrm{C}$ 条 件下恒温 $1 \mathrm{~h}$ 可以得到硫氧化物纳米板. 密度泛函理论 计算表明, 从轻稀土到重稀土, 其硫氧化物的热力学稳 定性逐渐降低, 形成氧化物的趋势逐渐增强. 本文中的 合成方法有效提高了反应体系中硫元素的化学势, 从而 实现了中重稀土硫氧化物纳米板的液相合成. 所合成的 $\mathrm{Y}_{2} \mathrm{O}_{2} \mathrm{~S}: \mathrm{Eu}$ 纳米板可以作为一种红色荧光纳米材料. 与 体相材料相比, 其荧光激发和发射光谱均受纳米尺寸下 的表面效应和缺陷态影响有所变化. 其苂光寿命较体相 材料短, 量子产率较体相材料低. 预期通过热处理提高 $\mathrm{Y}_{2} \mathrm{O}_{2} \mathrm{~S}: \mathrm{Eu}$ 纳米板的晶化程度或通过制备核壳结构可以
大幅度阻断表面弛豫过程对于苂光的淬灭，从而有望提 高这种纳米材料的苂光量子产率，并使其在显示、成像 等领域具有应用潜力.

\section{4 实验部分}

\section{1 稀土硫氧化物纳米板的合成}

\subsection{1 稀土乙酰丙酮盐的合成}

稀土(Y, Eu, Gd, Er 和 Yb)乙酰丙酮盐的合成方法由 参考文献[33]中的合成方法改进而来. 具体步骤为: 10 $\mathrm{mmol}$ 稀土氧化物 $\left(\mathrm{RE}_{2} \mathrm{O}_{3}\right)$ 与 $40 \mathrm{~mL}$ 去离子水混合, 搅拌 下加硝酸至溶解, 滤去不溶物. $8.01 \mathrm{~g}$ 乙酰丙酮与 $40 \mathrm{~mL}$ 去离子水混合, 搅拌下加氨水至不分相. 两份溶液混合 后滴加氨水至 $\mathrm{pH}$ 达到 6 7 之间，得沉淀. 减压过滤， 沉淀用去离子水洗涤两次, $60{ }^{\circ} \mathrm{C}$ 干燥过夜, 作为合成反 应的前驱体.

\subsection{2 稀土硫氧化物纳米板的合成}

将 $0.5 \mathrm{mmol}$ 稀土乙酰丙酮盐与 $10.78 \mathrm{~g}$ 油胺混合, $100{ }^{\circ} \mathrm{C}$ 下真空除气 $20 \mathrm{~min}$. 向油胺溶液中持续鼓入 $20 \%$ 硫化氢与 $80 \%$ 氮气的混合气体，气体流量为 60 $\mathrm{mL} \cdot \mathrm{min}^{-1}$. 将油酸溶液以 $20{ }^{\circ} \mathrm{C} \cdot \mathrm{min}^{-1}$ 的升温速度加热 到 $280{ }^{\circ} \mathrm{C}$, 并将其温度维持在 $280{ }^{\circ} \mathrm{C} 1 \mathrm{~h}$, 然后自然冷 却至室温.

待油胺溶液冷却后, 向其中加入 $50 \mathrm{~mL}$ 乙醇, 将混 合液超声处理至均匀，以 $7800 \mathrm{r} / \mathrm{min}$ 的速度离心 $10 \mathrm{~min}$, 弃去上清液. 沉淀用环己烷-乙醇混合溶剂洗涤, 离心 分离弃去上清液, 沉淀洗涤两次后真空干燥, 得到稀土 硫氧化物纳米板粉末. 该粉末可分散于环己烷、甲苯、 氯仿中.

在反应原料中加入 $0.302 \mathrm{~g}$ 油酸钠, 采用相同的合 成方法, 可以合成得到钠掺杂的稀土硫氧化物纳米板.

以 $0.48 \mathrm{mmol}$ 乙酰丙酮钎和 $0.02 \mathrm{mmol}$ 乙酰丙䣶铕 作为稀土前驱体，采用相同的合成方法，可以合成得到 $\mathrm{Y}_{2} \mathrm{O}_{2} \mathrm{~S}: \mathrm{Eu}$ 纳米板.

\section{2 前驱体及硫氧化物纳米板的结构及性质表征}

所有样品的 TEM 表征在 JEM-2100F 型场发射高分 辨透射电子显微镜(JEOL, 日本)上进行, 加速电压为 $200 \mathrm{kV}$ ，并配备了 EDS 表征组件. XRD 表征在 D/max-2000 型 X 射线粉末衍射仪(Rigaku, 日本)上进行, 使用的 $\mathrm{X}$ 射线为 $\mathrm{Cu}-\mathrm{K} \alpha$ 线，管压为 $40 \mathrm{kV}$, 管流为 100 $\mathrm{mA}$, 扫描速度为 $4\left(^{\circ}\right) \cdot \mathrm{min}^{-1}$, 狭缝宽度为 $1 / 2$. 乙酰丙酮 钎的热重分析在 V2.60 TA 热重差热分析仪(Universal, 美国)上进行, 样品在氮气中以 $5{ }^{\circ} \mathrm{C} \cdot \mathrm{min}^{-1}$ 的速度从室 温升温至 $1200{ }^{\circ} \mathrm{C}$, 所用参照物为 $\alpha-\mathrm{Al}_{2} \mathrm{O}_{3} . \mathrm{Y}_{2} \mathrm{O}_{2} \mathrm{~S}: \mathrm{Eu}$ 纳 米板环已烷分散液及其体相材料的荧光光谱表征在 F-4500 型荧光光谱仪(Hitachi, 日本)上进行, 光源为氙 灯, 其功率为 $150 \mathrm{~W}$, 带通为 $0.2 \mathrm{~nm}$, 在室温下测试, 激 发和发射狭缝宽度均为 $2.5 \mathrm{~nm}$. 苂光寿命表征在 
FLS920 型瞬态稳态苂光光谱仪(Edinburgh Instruments Ltd., 英国)上完成, 测试温度为室温. PLQY 测定在 Nanolog FL3-2iHR 型红外苂光综合测试系统(Horiba Jobin Yvon, 法国)上完成.

\section{3 稀土硫氧化物及氧化物生成能的 DFT 计算}

钎和镥的硫氧化物及氧化物的生成能的密度泛函 理论计算在 Vienna Ab-initio Simulation Package (VASP, version 5.2) [34] $^{[34}$ 进行. 采用广义梯度近似(generalized gradient approximation, GGA), 交换关联泛函采用 Perdew-Burke-Ernzerhof (PBE) 泛函 ${ }^{[35]}$, 芯层电子按投影 缀加波方法处理(projected augmented wave, PAW) ${ }^{[36]}$. 所采用的平面波基组的截断能为 $500 \mathrm{eV}$. 计算过程为首 先进行离子结构弛豫, 优化晶胞参数及原子坐标, 然后 对优化后结果进行单点能计算. 结构优化时离子结构弛 豫的收玫条件为两次能量差低于 $10^{-3} \mathrm{eV}$, 电子密度自 洽场运算的收敛条件为两次能量差低于 $10^{-4} \mathrm{eV}$; 单点 能计算时电子密度自洽场运算的收玫条件为两次能量 差低于 $10^{-5} \mathrm{eV}$. 对于 $\mathrm{Y}_{2} \mathrm{O}_{2} \mathrm{~S}$ 和 $\mathrm{Lu}_{2} \mathrm{O}_{2} \mathrm{~S}$, 计算使用的是六 方晶胞, 空间群为 $P-3 m l\left(\mathrm{Y}_{2} \mathrm{O}_{2} \mathrm{~S}\right.$ : JCPDS 24-1424, $a=$ $3.784 \AA$, $c / a=1.741, Z=1 ; \mathrm{Lu}_{2} \mathrm{O}_{2} \mathrm{~S}$ : JCPDS 26-1445, $a=$ $3.707 \AA, c / a=1.751, Z=1)$, 布里渊区中的 $k$ 点取法采用 $5 \times 5 \times 3$ 的 Monkhorst-Pack 网格. 对于 $\mathrm{Y}_{2} \mathrm{O}_{3}$ 和 $\mathrm{Lu}_{2} \mathrm{O}_{3}$, 计算使用的是立方晶胞, 空间群为 $I a-3\left(\mathrm{Y}_{2} \mathrm{O}_{3}\right.$ : JCPDS 25-1200, $a=10.604 \AA, Z=16 ; \mathrm{Lu}_{2} \mathrm{O}_{3}$ : JCPDS 12-0728, $a=10.39 \AA, Z=16$ ), 布里渊区中的 $k$ 点取法采用 $3 \times 3 \times 3$ 的 Monkhorst-Pack 网格. 此外, 钇、镥、氧和 硫单质的单点能也使用该软件进行了计算, 结果用于计 算硫氧化物和氧化物的生成能. 但是单质能量的计算结 果会在比较不同稀土元素硫氧化物和氧化物的稳定性 时消去，因而不会对比较结果产生影响.

\section{References}

[1] Wang, F.; Liu, X. Chem. Soc. Rev. 2009, 38, 976.

[2] Feng, W.; Sun, L.-D.; Zhang, Y.-W.; Yan, C.-H. Coord. Chem. Rev. 2010, 254, 1038 .

[3] Wang, G.; Peng, Q.; Li, Y. Acc. Chem. Res. 2011, 44, 322.

[4] Stouwdam, J. W.; Hebbink, G. A.; Huskens, J.; van Veggel, F. C. J. M. Chem. Mater. 2003, 15, 4604.

[5] Liu, L.; Chen, X. Nanotechnology 2007, 18, 255704.
[6] Wang, F.; Banerjee, D.; Liu, Y.; Chen, X.; Liu, X. Analyst 2010 , $135,1839$.

[7] Chen, Z.; Chen, H.; Hu, H.; Yu, M.; Li, F.; Zhang, Q.; Zhou, Z.; Yi, T.; Huang, C. J. Am. Chem. Soc. 2008, 130, 3023.

[8] Zhou, J.; Sun, Y.; Du, X.; Xiong, L.; Hu, H.; Li, F. Biomaterials 2010, 31, 3287.

[9] Wang, R.; Crozier, P. A.; Sharma, R.; Adams, J. B. Nano Lett. 2008, 8,962 .

[10] Mai, H.-X.; Sun, L.-D.; Zhang, Y.-W.; Si, R.; Feng, W.; Zhang, H.-P.; Liu, H.-C.; Yan, C.-H. J. Phys. Chem. B 2005, 109, 24380.

[11] Selinsky, R. S.; Han, J. H.; Morales Pérez, E. A.; Guzei, I. A.; Jin, S. J. Am. Chem. Soc. 2010, 132, 15997.

[12] Zhao, F.; Sun, H.-L.; Su, G.; Gao, S. Small 2006, 2, 244.

[13] Si, R.; Zhang, Y.-W.; You, L.-P.; Yan, C.-H. Angew. Chem. Int. Ed. 2005, 44, 3256

[14] Wang, D.; Wang, Z.; Zhao, P.; Zheng, W.; Peng, Q.; Liu, L.; Chen, X.; Li, Y. Chem. Asian J. 2010, 5, 925.

[15] Zhang, Y.-W.; Sun, X.; Si, R.; You, L.-P.; Yan, C.-H. J. Am. Chem. Soc. 2005, 127, 3260 .

[16] Wang, X.; Zhuang, J.; Peng, Q.; Li, Y. D. Inorg. Chem. 2006, 45, 6661 .

[17] Du, Y.-P.; Zhang, Y.-W.; Yan, Z.-G.; Sun, L.-D.; Yan, C.-H. J. Am. Chem. Soc. 2009, 131, 16364

[18] Mai, H.-X.; Zhang, Y.-W.; Si, R.; Yan, Z.-G.; Sun, L.-D.; You, L.-P.; Yan, C.-H. J. Am. Chem. Soc. 2006, 128, 6426.

[19] Boyer, J.-C.; Cuccia, L. A.; Capobianco, J. A. Nano Lett. 2007, 7, 847.

[20] Li, C.; Quan, Z.; Yang, J.; Yang, P.; Lin, J. Inorg. Chem. 2007, 46, 6329.

[21] Zhang, F.; Li, J.; Shan, J.; Xu, L.; Zhao, D. Y. Chem. Eur. J. 2009, 15, 11010 .

[22] Du, Y.-P.; Zhang, Y.-W.; Sun, L.-D.; Yan, C.-H. J. Am. Chem. Soc. 2009, 131, 3162 .

[23] Ding, Y.; Gu, J.; Ke, J.; Zhang, Y.-W.; Yan, C.-H. Angew. Chem. Int. Ed. 2011, 50, 12330.

[24] Ding, Y.; Gu, J.; Zhang, T.; Yin, A.-X.; Yang, L.; Zhang, Y.-W.; Yan, C.-H. J. Am. Chem. Soc. 2012, 134, 3255.

[25] Zhao, F.; Yuan, M.; Zhang, W.; Gao, S. J. Am. Chem. Soc. 2006 $128,11758$.

[26] Wang, X.; Zhang, Z.; Tang, Z.; Lin, Y. Mater. Chem. Phys. 2003, 80,1 .

[27] Dai, Q.; Song, H.; Wang, M.; Bai, X.; Dong, B.; Qin, R.; Qu, X.; Zhang, H. J. Phys. Chem. C 2008, 112, 19399.

[28] Dhanaraj, J.; Jagannathan, R.; Trivedi, D. C. J. Mater. Chem. 2003, 13,1778 .

[29] Liu, G.; Conn, C. E.; Drummond, C. J. J. Phys. Chem. B 2009, 113, 15949.

[30] Thomson, J. W.; Nagashima, K.; Macdonald, P. M.; Ozin, G. A. J. Am. Chem. Soc. 2011, 133, 5036.

[31] Pearson, R. G. J. Am. Chem. Soc. 1963, 85, 3533.

[32] Huignard, A.; Gacoin, T.; Boilot, J.-P. Chem. Mater. 2000, 12, 1090.

[33] Stites, J. G.; McCarty, C. N.; Quill, L. L. J. Am. Chem. Soc. 1948, 70,3142

[34] Kresse, G.; Furthmuller, J. Phys. Rev. B 1996, 54, 11169.

[35] Perdew, J. P.; Burke, K.; Ernzerhof, M. Phys. Rev. Lett. 1996, 77, 3865.

[36] Blochl, P. E. Phys. Rev. B 1994, 50, 17953. 